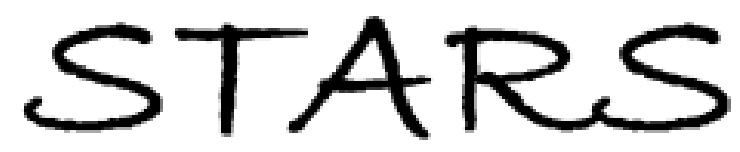

University of Central Florida

STARS

$1-1-2009$

\title{
Effect of hydration layer and surface wettability in enhancing thermal conductivity of nanofluids
}

\author{
Parveen Sachdeva \\ University of Central Florida \\ Ranganathan Kumar \\ University of Central Florida
}

Find similar works at: https://stars.library.ucf.edu/facultybib2000 University of Central Florida Libraries http://library.ucf.edu

This Article is brought to you for free and open access by the Faculty Bibliography at STARS. It has been accepted for inclusion in Faculty Bibliography 2000s by an authorized administrator of STARS. For more information, please contact STARS@ucf.edu.

\section{Recommended Citation}

Sachdeva, Parveen and Kumar, Ranganathan, "Effect of hydration layer and surface wettability in enhancing thermal conductivity of nanofluids" (2009). Faculty Bibliography 2000s. 2077.

https://stars.library.ucf.edu/facultybib2000/2077

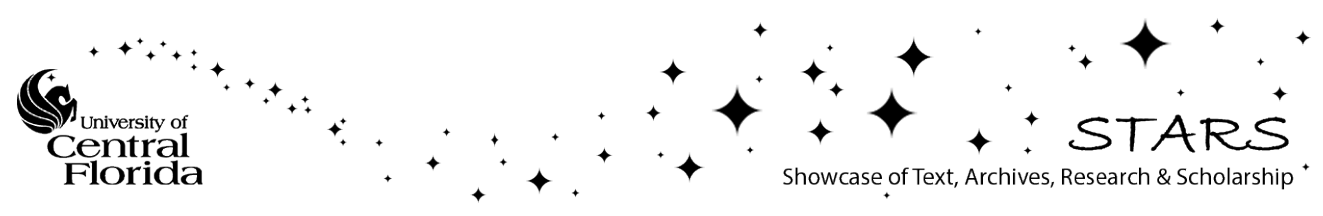




\section{Effect of hydration layer and surface wettability in enhancing thermal conductivity of nanofluids}

Cite as: Appl. Phys. Lett. 95, 223105 (2009); https://doi.org/10.1063/1.3270003

Submitted: 04 August 2009 . Accepted: 10 November 2009 . Published Online: 02 December 2009

Parveen Sachdeva, and Ranganathan Kumar

\section{ARTICLES YOU MAY BE INTERESTED IN}

Molecular dynamics simulation of effective thermal conductivity and study of enhanced thermal transport mechanism in nanofluids

Journal of Applied Physics 102, 074302 (2007); https://doi.org/10.1063/1.2785009

Role of Brownian motion in the enhanced thermal conductivity of nanofluids Applied Physics Letters 84, 4316 (2004); https://doi.org/10.1063/1.1756684

Role of Brownian motion hydrodynamics on nanofluid thermal conductivity Applied Physics Letters 88, 093116 (2006); https://doi.org/10.1063/1.2179118

\section{Applied Physics Letters}

Mid-IR and THz frequency combs special collection 


\title{
Effect of hydration layer and surface wettability in enhancing thermal conductivity of nanofluids
}

\author{
Parveen Sachdeva and Ranganathan Kumar ${ }^{\mathrm{a})}$ \\ Department of Mechanical Materials and Aerospace Engineering, University of Central Florida, Orlando, \\ Florida 32816, USA
}

(Received 4 August 2009; accepted 10 November 2009; published online 2 December 2009)

\begin{abstract}
In a molecular dynamics study of water based nanofluids, we show that a hydration layer is formed at the particle-fluid interface, where the attraction or cohesive potential between the liquid molecules is dominant. In the hydration layer, the collision mode rather than the kinetic or potential mode is primarily responsible for the enhancement of thermal transport properties in nanofluids, as it results in more interactions. The thermal conductivity for a wetting particle is found to be higher than for a neutral or a nonwetting particle. (c) 2009 American Institute of Physics. [doi:10.1063/1.3270003]
\end{abstract}

The interface between a solid and liquid or solid and solid is a study of great interest since the surface can either provide thermal barrier or thermal enhancement. The interfacial resistance, also called the Kapitza resistance, arises due to the difference in phonon spectra between the two phases. Nanocrystalline materials with large Kapitza resistance and grain boundaries less than $40 \mathrm{~nm}$ can decrease the thermal conductivity severalfold and provide a thermal barrier. ${ }^{1}$ Quite the opposite application of enhancing thermal conductivity can be found in nanofluids. When nanoparticles are suspended in water, a hydration layer is formed around each particle. When there is a strong bonding between the solid and liquid atoms at the interface, the Kapitza resistance is lower. The hydration layer scatters the incoming and the outgoing phonons, and influences the interface characteristics, and increases the nanofluid thermal conductivity. Molecular dynamics (MD) study has shown evidence of the hydration layer; ${ }^{2,3}$ however, there was no conclusive evidence from their work that the altered thermal transport properties of the layered liquid caused the enhancement in thermal conductivity. Earlier, ${ }^{4}$ we showed that Brownian motion accounts for up to $6 \%$ increase in thermal conductivity by random walk motion and not diffusion, and that this increase is limited by the maximum concentration and is less than that predicted by effective medium theory. Beyond the maximum limit, we showed that particle aggregates begin to form, as originally proposed using Monte Carlo simulations. ${ }^{5}$ The objective of this work is to revisit the hydration layer theory and confirm its existence through a MD simulation and offer reasons for its contribution to higher conductivity. The second objective is to understand the effects of surface wettability of nanoparticle on the thermal conductivity of nanofluids.

Previous studies $^{6-9}$ involving MD simulation of nanofluids have considered simplistic Lennard-Jones (LJ) potential to model the interactions between solid-solid, liquidliquid, and solid-liquid atom pairs. Table I shows a list of the MD simulation studies performed on nanofluids and the interaction potentials used in each of them. ${ }^{6-11}$ In this work, liquid water is modeled as a flexible bipolar molecule using the flexible 3 center (F3C) model. ${ }^{12}$ This model maintains the tetrahedral structure of the water molecule and allows the

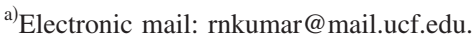

$\mathrm{H}-\mathrm{O}-\mathrm{H}$ bond bending and $\mathrm{O}-\mathrm{H}$ bond stretching modes, thereby mimicking the motion and interactions between real water molecules. As a first step, a simple two body LJ potential is used to model the solid nanoparticle. In addition, each atom in the nanoparticle is connected to its first neighbors by finite extensible nonlinear elastic (FENE) bonding potential, ${ }^{11} U_{\mathrm{FENE}}=(k / 2) R_{o}^{2} \ln \left[1-\left(r / R_{o}\right)^{2}\right], r<R_{o}$, where $R_{o}=1.5 \sigma$ and $k=30.0 \varepsilon / \sigma^{2}$. The LJ parameters ${ }^{8}$ of $\mathrm{Cu}$ are used for the solid particle. A simple two body LJ potential is used to model the solid-fluid interactions, with the LJ parameters calculated from the Lorentz-Berthelot mixing rule. ${ }^{13}$ The thermal conductivity is calculated by combining the linear response theory with equilibrium molecular dynamic simulation (Green-Kubo formulation ${ }^{13}$ ). The thermal conductivity is calculated using the time integral of the heat current autocorrelation function, $\lambda$ $=1 / 3 k_{B} V T^{2} \int_{0}^{\infty}\langle\vec{q}(0) \cdot \vec{q}(t)\rangle d t$, where $\lambda$ is the thermal conductivity, $V$ is the system volume, $T$ is the system temperature, $k_{B}$ is the Boltzmann constant, and $\vec{q}$ is the instantaneous heat flux ${ }^{14}$ vector as given by

$$
\begin{aligned}
\vec{q}(t)= & \sum_{j} \vec{v}_{j}\left(\frac{1}{2} m_{i} v_{i}^{2}+\frac{1}{2} \sum_{i} \phi_{i j}\left(r_{i j}\right)\right)+\frac{1}{2} \sum_{i j, i \neq j} \vec{r}_{i j}\left(\vec{f}_{i j} \cdot \vec{v}_{i}\right) \\
& -\sum_{j} \vec{v}_{j} h .
\end{aligned}
$$

The third term on the right hand side consisting of the mean (instantaneous) enthalpy ( $\boldsymbol{h})$ arises because of the definition of the heat current in a binary system, where heat and mass are coupled. The mean enthalpy $(\boldsymbol{h})$ is calculated as the sum of average potential energy, average kinetic energy, and av-

TABLE I. MD simulation studies of nanofluid (SS, LL, and SL refer to

\begin{tabular}{|c|c|c|c|c|}
\hline & Ref. & Potential used & $\begin{array}{c}\text { Volume } \\
\text { concentration }\end{array}$ & $\begin{array}{l}\text { Conductivity } \\
\text { enhancement }\end{array}$ \\
\hline 1 & 6 & $\mathrm{LJ}$ for $\mathrm{SS}, \mathrm{LL}, \mathrm{SL}$ & $10 \%$ & $\cdots$ \\
\hline 2 & 7 & LJ for SS, LL, SL (Xe-Pt) & $0.8 \%$ & $35 \%$ \\
\hline 3 & 8 & $\mathrm{LJ}$ for $\mathrm{SS}, \mathrm{LL}, \mathrm{SL}(\mathrm{Ar}-\mathrm{Cu})$ & $8 \%$ & $52 \%$ \\
\hline 4 & 9 & LJ for SS, LL, SL (Ar-Cu) & $1.5 \%$ & $\cdots$ \\
\hline 5 & 10 & LJ-FENE for SS, LJ for LL, SL & $3.3 \%$ & $2.5 \%$ \\
\hline 6 & 11 & LJ-FENE for SS, LJ for LL, SL & $\cdots$ & $\cdots$ \\
\hline
\end{tabular}
solid-solid, liquid-liquid, and solid-liquid, respectively). 


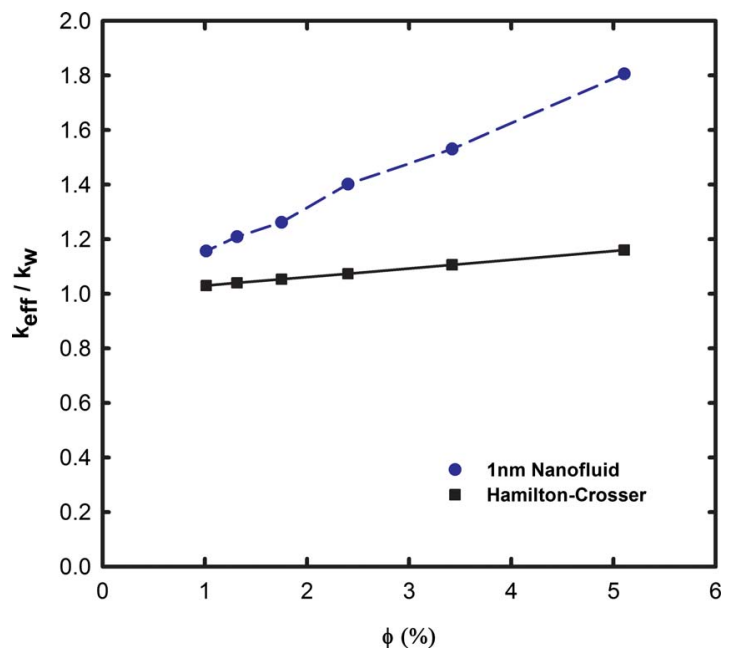

FIG. 1. (Color online) Thermal conductivity vs volume concentration for $1 \mathrm{~nm}$ particle.

erage virial terms per particle of each species.

To validate the F3C model, 216, 343, 512, 729, and 1000 water molecules were used in a series of simulation at $300{ }^{\circ} \mathrm{K}$ temperature and $1 \mathrm{~atm}$. pressure. A good match with the experimental thermal conductivity of $0.61 \mathrm{~W} / \mathrm{m} \mathrm{K}$ was obtained for these runs. The second step in the validation involves the calculation of thermal conductivity of liquid argon which was compared with that of Vogelsang et al. ${ }^{15}$ at state point defined by the dimensionless temperature and number density as $T^{*}=0.73$ and $\rho^{*}=0.8442$. The MD tool was separately validated for liquid argon and water only systems and an excellent match in thermal conductivity was found between calculations and measurements.

The simulation domain in our MD simulation consists of one nanoparticle surrounded by tetrahedral water molecules. Periodic boundary conditions are applied in all directions which implicitly conserve mass and momentum in a MD simulation and mimic a three-dimensional nanofluid system. The $1 \mathrm{~nm}$ nanoparticle consists of 44 atoms and the $2 \mathrm{~nm}$ nanoparticle consists of 360 atoms. The nanofluid simulations were run at a system temperature of $300{ }^{\circ} \mathrm{K}$ and pressure of $1 \mathrm{~atm}$, and the simulation domain size was varied with the number of water molecules to maintain a density of $1 \mathrm{~g} / \mathrm{cm}^{3}$. MD time step of $0.1 \mathrm{fs}$ was used and fifth order predictor-corrector method was used to integrate the equations of motion.

The system was first equilibrated in the $N V T$ ensemble for $5 \times 10^{5}$ time steps. In the equilibration period, the system temperature was kept constant using the velocity scaling method. After the equilibration period, the temperature constraint was removed and the system was allowed to evolve in the $N V E$ ensemble for $10^{6}$ time steps. In this period, the heat current was calculated and the Green-Kubo correlation was used to calculate the thermal conductivity of the nanofluid system. The effect of particle volume fraction on nanofluid thermal conductivity is presented in Fig. 1. The thermal conductivity increases almost linearly from 0.705 to $1.101 \mathrm{~W} / \mathrm{m} \mathrm{K}$ for concentration from $1 \%$ to $5.1 \%$ for the 1 $\mathrm{nm}$ particle, as seen in previous studies. ${ }^{7,8}$

The total heat flux is written as the sum of three terms of kinetic, potential, and collision ${ }^{7}$ as $\vec{q}=\vec{q}_{K}+\vec{q}_{P}+\vec{q}_{C}$. From the heat current autocorrelation function in the Green-Kubo correlation, this will give rise to three main modes of thermal

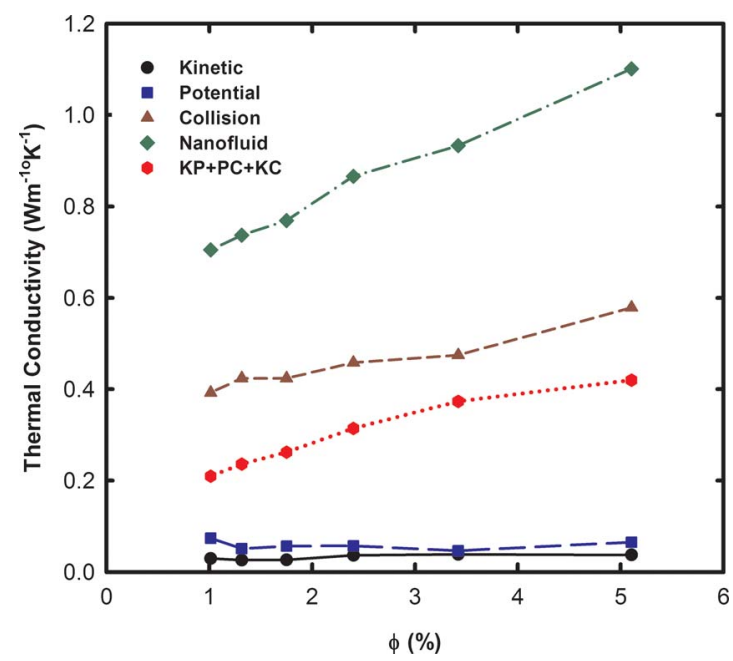

FIG. 2. (Color online) Thermal conductivity contribution by various heat current modes.

conductivity, namely, $\mathrm{KK}, \mathrm{PP}$, and $\mathrm{CC}$, along with the cross components, indicated by KP, PC, and KC (Fig. 2). Collision is the dominant mode of the thermal conductivity due to the motion of the water molecules that are densely packed around the particle. This collision mode is also responsible for increasing the thermal conductivity at higher concentrations. This observation is in contrast to the observation of Eapen et $a l^{7}$ that the increase in thermal conductivity with increasing volume fraction comes from the potential mode, arising from the strong cross interaction between $\mathrm{Xe}$ and $\mathrm{Pt}$. In our study, Lorentz-Berthelot mixing rule was used to calculate the LJ parameters for the solid-liquid cross interaction.

The motion and interaction of the liquid and solid atoms at the atomic scale is the cause of all macroscale properties of any material, including the enhanced thermal conductivity of nanofluids. By using the atomic position data at various time steps the mean square displacement (MSD) can be calculated for the liquid and solid molecules. Comparison of the MSD of liquid molecules in the base fluid and in the nanofluid can give an insight into the thermal transport mechanism in nanofluids. MSD of liquid molecules in the $1 \%$ nanofluid is approximately 1.1 times higher compared with that of the base fluid. MSD of liquid atoms in nanofluid at various volume concentrations is consistently higher compared with the base fluid. An increase in MSD and diffusion coefficient was reported in the presence of nanoparticles without the hydration layer formation. ${ }^{8}$ Both MSD and thermal conductivity increase with increasing volume fraction of the nanoparticle. MSD for various nanofluids shows that there is a significant increase in the movement, and hence the displacement of the liquid molecules due to the presence of solid nanoparticle. This enhanced movement will eventually mean that the liquid molecules undergo more frequent interactions, and hence transport more energy. This is accentuated by the fact that there are additional liquid molecules close to the nanoparticle (Fig. 3). Since these liquid molecules are more closely packed than other liquid molecules away from the particle, the attraction or cohesive potential between the liquid molecules is dominant. This further establishes the fact that the collision mode in the thermal conductivity is dominant in nanofluids.

This layer of liquid molecules at the particle-fluid interface is also called the hydration layer. The density distribu- 


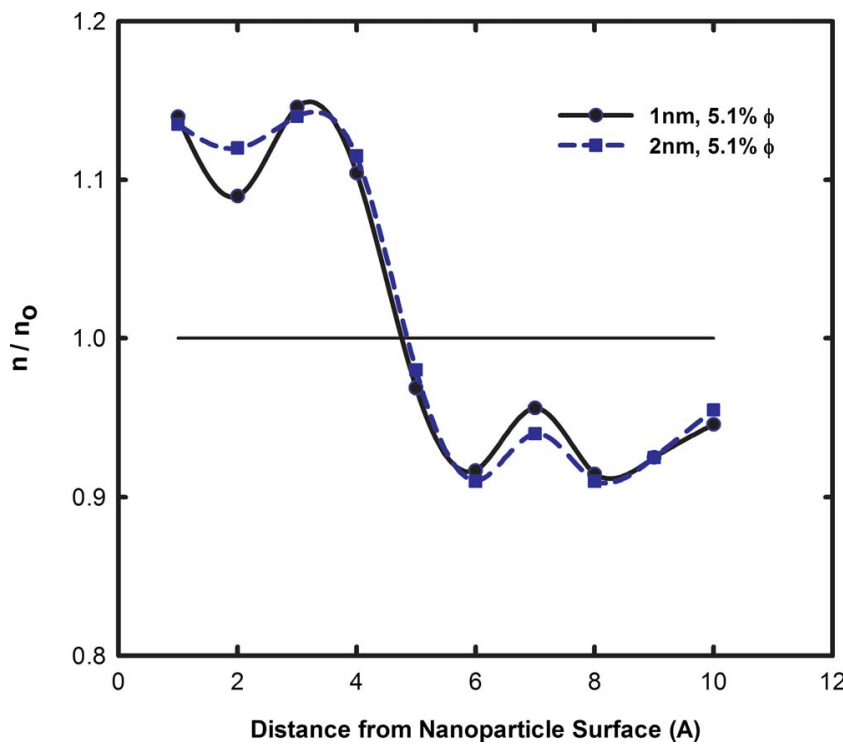

FIG. 3. (Color online) Relative number density distribution around the nanoparticle as a function of distance from the nanoparticle surface.

tion of liquid atoms around the nanoparticle using the trajectory data shows the presence of the hydration layer (Fig. 3). The simulation domain is divided into multiple concentric spherical shells around the nanoparticle and the number density of liquid molecules is calculated in these spherical shells. The density of water is high near the surface of the nanoparticle at least up to $0.5 \mathrm{~nm}$ away for both 1 and $2 \mathrm{~nm}$ particles, which is consistent with the observation made by Li et al. ${ }^{9}$ High MSD of water molecules near the particle surface is due to increased interaction between the solid and liquid atoms near the surface, which results in higher transport of energy in the nanofluid.

The interactions we have considered thus far are for neutral surfaces. In order for nanoparticles to stay in colloidal suspension, chemical dispersants are used. To get further insight into the particle behavior, the effect of surface wettability of a nanoparticle is studied next. To understand this effect, the strength of the attractive force between the solid and fluid atoms is varied. The $\mathrm{cr}^{-6}$ term in the LJ potential determines the attraction force between a pair of atoms, and varying this constant will vary the strength of solid-liquid attraction force: ${ }^{11} u_{s f}=4 \varepsilon_{s f}\left[(\sigma / r)^{12}-c(\sigma / r)^{6}\right]$. A value of $c$ $<1$ would indicate less attraction force between solid-liquid atoms for a nonwetting particle, and $c<1$ indicates a higher attraction force for a highly wetting particle. The value $c$ is varied from 0.25 to 1.25 in this study to model a range of wetting properties and to simulate varying Kapitza resistance at the solid-liquid interface. High $c$ translates to lower Kapitza resistance and a low $c$ value gives a higher Kapitza resistance. ${ }^{11}$ The nanofluid thermal conductivity increases as

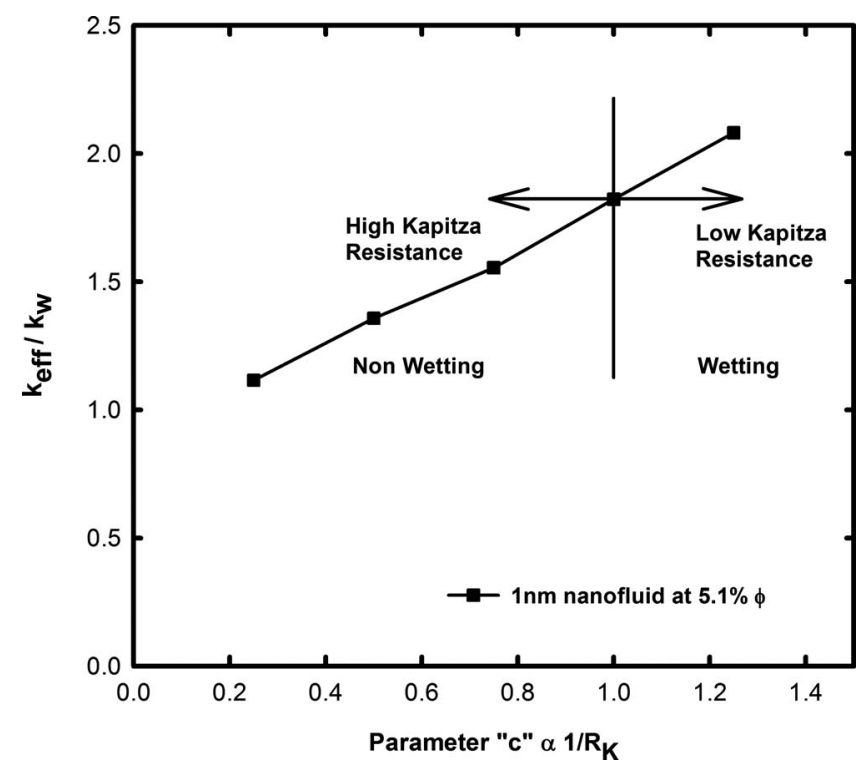

FIG. 4. Thermal conductivity vs particle wettability (increasing $c$ indicates higher wettability).

$c$ is increased, i.e., the thermal conductivity is higher for a wetting particle compared with a nonwetting particle (Fig. 4). When there is a strong interaction between the solid and liquid atoms at the interface, the Kapitza resistance is lower, ${ }^{11}$ and it creates a dynamic interface around the nanoparticle that facilitates the exchange of energy between solid and liquid atoms.

The authors acknowledge Dr. Pawel Keblinski and Dr. William Evans for their helpful discussions with implementation of the $\mathrm{F} 3 \mathrm{C}$ model for water.

${ }^{1}$ J. A. Eastman, H. S. Yang, L. J. Thompson, and G. R. Bai, http:// chemistry.anl.gov/msdreview/complex_oxides/kapitzaysz.pdf, 2006.

${ }^{2}$ G. Cicero, J. C. Grossman, A. Catellani, and G. Galli, J. Am. Chem. Soc. 127, 6830 (2005).

${ }^{3}$ J. Eapen, J. Li, and S. Yip, Phys. Rev. E 76, 062501 (2007).

${ }^{4}$ A. Gupta and R. Kumar, Appl. Phys. Lett. 91, 223102 (2007).

${ }^{5}$ R. S. Prasher, W. Evans, P. Meakin, J. Fish, P. Phelan, and P. Keblinski, Appl. Phys. Lett. 89, 143119 (2006).

${ }^{6}$ P. Keblinski, S. R. Philpot, S. U. S. Choi, and J. A. Eastman, Int. J. Heat Mass Transfer 45, 855 (2002).

${ }^{7}$ J. Eapen, J. Li, and S. Yip, Phys. Rev. Lett. 98, 028302 (2007)

${ }^{8}$ S. Sarkar and R. P. Selvam, J. Appl. Phys. 102, 074302 (2007).

${ }^{9}$ L. Li, Y. Zhang, H. Ma, and M. Yang, Phys. Lett. A 372, 4541 (2008).

${ }^{10}$ W. Evans, J. Fish, and P. Keblinski, Appl. Phys. Lett. 88, 093116 (2006).

${ }^{11}$ M. Vladkov and J. L. Barrat, Nano Lett. 6, 1224 (2006).

${ }^{12}$ M. Levitt, M. Hirshberg, R. Sharon, K. E. Laidig, and V. Daggett, J. Phys. Chem. B 101, 5051 (1997).

${ }^{13}$ M. P. Allen and D. J. Tildesley, Computer Simulation of Liquids (Oxford Science, Oxford, 1987).

${ }^{14} \mathrm{C}$. Hoheisel, Theoretical Treatment of Liquids and Mixtures (Elsevier, New York, 1987).

${ }^{15}$ R. Vogelsang, C. Hoheisel, and G. Ciccotti, J. Chem. Phys. 86, 6371 (1987). 